Results A one standard deviation (SD) increase in weight gain between 7 and 15 years was associated with 0.50 years $(95 \%$ CI: $0.21,0.80$ ) higher AgeAccelGrim and, with 0.22 years (95\% CI: -0.11, 0.55) higher AgeAccelLevine. This was replicated in NCDS. For linear growth, there was some evidence that more rapid growth between 2 and 4 years was associated with lower AgeAccelLevine (-0.39 years [95\% CI: -0.74, $0.050)$ and AgeAccelGrim (-0.24 years [95\% CI: $-0.54,0.06])$. There was no evidence that relative weight gain and linear growth during childhood was associated with any other AgeAccel biomarker. There was no relationship between pubertal timing in men and any of the AgeAccel biomarkers at 53 years. Women who reached menarche $\geq 12$ years had 1.20 years $(95 \%$ CI: $0.15,2.24)$ higher AgeAccelGrim on average than women who reached menarche $<12$ years; however this was not replicated in NCDS.

Conclusion Our findings support the use of the second generation DNAm age biomarkers as markers of ageing and reinforces the idea that faster gains in weight during adolescence has lasting implications for healthy ageing.

\section{P05 PERFORMANCE ON DIFFERENT COGNITIVE TESTS PREDICT FUTURE DEMENTIA: FIFTEEN YEARS OF FOLLOW-UP IN A BRITISH COHORT STUDY}

SA Hayat*, R Luben, KT Khaw, C Brayne. Public Health and Primary Care, University of Cambridge, Cambridge, UK

\subsection{6/jech-2020-SSMabstracts. 101}

Background Current policies do not support screening for future dementia among apparently healthy individuals. This is due to the lack of certainty of the clinical outcome and effective interventions. Numerous dementia risk models have been developed, currently, all within the research setting only. Studies have shown impairment in multiple cognitive domains several years before a clinical diagnosis of dementia.

Methods We examined the utility of an extensive battery assessing a range of specific cognitive abilities as well as a composite global score, to predict dementia. Dementia outcomes were ascertained using electronic health record linkage in 8581 individuals (aged 48-92 years) taking part in the EPIC-Norfolk study. Participants were followed for 15 years (2004-2019). Risk of dementia was estimated using Cox proportional hazard models adjusting for sociodemographic, lifestyle and health variables, evaluating discriminative accuracy of the models by analysing receiver operating characteristic (ROC) curves.

Results Poor cognition was predictive of incident dementia, even after adjustment for co-variates. Those with a poor performance score for global cognition (bottom 10\%) were almost four times as likely to get a dementia diagnosis than those who performed well $(\mathrm{HR}=3.51$ (95\%CI 2.61, 4.71) $\mathrm{P}<0.001)$. Associations were observed for specific as well as global cognitive abilities. The test for episodic (verbal) memory outperformed other tests and was comparable to global cognition scores. Poor cognition in four or more tests was associated with 10 -fold increased risk of developing dementia compared to those not performing poorly in any test $(\mathrm{HR}=10.82(95 \%$ CI $6.85,17.10) \mathrm{P}<0.001))$. Cognitive measures strengthen prediction models of dementia (AUC $=0.85$ (95\%CI 0.82, 0.87) $\mathrm{P}<0.001)$.

Discussion This study provides further insight on poor cognition predicting future dementia. This association was observed for global cognition and specific abilities, particularly for verbal episodic memory. Impairment occurs in multiple domains several years prior to a clinical diagnosis, and the more pervasive and greater the variability, the higher the risk of dementia. Deficits across multiple domains predict over and above individual test scores.

\section{P06 SCHIZOPHRENIA POLYGENIC RISK PREDICTS GENERAL COGNITIVE DEFICIT BUT NOT FURTHER COGNITIVE DECLINE IN HEALTHY OLDER ADULTS}

1,20 Ainakina*, ${ }^{3}$ A Kẹpińska, ${ }^{3} \mathrm{~J}$ MacCabe, ${ }^{1} \mathrm{D}$ Cadar, ${ }^{1} \mathrm{~A}$ Steptoe, ${ }^{3} \mathrm{R}$ Murray. ${ }^{1}$ Department of Behavioural Science and Health, Institute of Epidemiology and Health Care, University College London, London, UK; ${ }^{2}$ Department of Biostatistics and Health Informatics, Institute of Psychiatry, Psychology and Neuroscience, King's College London, University of London, London, UK; ${ }^{3}$ Department of Psychosis Studies, Institute of Psychiatry, Psychology and Neuroscience, King's College London, London, UK

\subsection{6/jech-2020-SSMabstracts. 102}

Background There has been a long argument over whether or not schizophrenia is a neurodegenerative disorder associated with progressive brain changes and increasing cognitive impairment. Building on the fact that schizophrenia is highly heritable and overlaps genetically with general cognitive ability, we investigated whether common genetic variants associated with schizophrenia additively confer a stable deficit in cognitive ability, a greater risk of cognitive decline over time, or both, over the 8-year follow-up, independently from the effects of the apolipoprotein $\mathrm{E}$ gene (APOE- $\epsilon 4$ ), in phenotypically welldefined sample of healthy older adults.

Methods We used data from the English Longitudinal Study of Ageing study which encompassed 6817 population-representative English adults who were followed-up for 8 years. Cognitive function was measured with well-established tests of memory (tests of immediate and delayed word recall combined into a single measure of correctly recalled words) and executive function (a test of verbal fluency where participants name as many animals as possible in a minute). Polygenic score for schizophrenia (SZ-PGS) was calculated based on the results from Psychiatric Genomics Consortium. Linear mixed effect models with maximum likelihood estimation were used to estimate baseline status and rate of change in cognition associated with SZ-PGS.

Results The sample baseline mean age was 64.3 years old (standard deviation $(\mathrm{SD})=9.3, \quad$ range $=50-101) ; \quad 25.3 \%$ $(\mathrm{N}=1724)$ of participants were carriers of APOE- $\epsilon 4$ and $46.2 \%(\mathrm{~N}=3159)$ were men. The average baseline memory score was $10.4(\mathrm{SD}=3.4)$ and executive function score was 20.7 (SD=6.3). One standard deviation increase in SZ-PGS was associated with a lower baseline executive function score (-0.23, 95\%CI-0.38 - -0.08) but not memory. SZ-PGS was not associated with rates of change in these cognitive domains during the 8-year follow-up period. However, APOE- $\epsilon 4$, tobacco smoking and lower wealth were associated with a decrease in the rate of memory and executive function during follow-up.

Discussion Common genetic variants associated with schizophrenia additively confer a stable deficit in cognitive ability but not cognitive ageing. The fact that we observed cognitive decline in our sample over the 8-year follow-up, associated with of APOE- $\epsilon 4$, tobacco smoking and lower wealth demonstrates that our study had the capacity to show cognitive 
decline. Therefore, we may conclude that while genetic susceptibility to schizophrenia conveys developmental cognitive deficit, it does not result in an ongoing cognitive decline, at least in later life. This, in turn, disproves the Kraepelinan notion of schizophrenia as a genetically determined progressively deteriorating brain disease.

\section{P07 LONG-TERM TRENDS IN LUNG CANCER INCIDENCE IN UK NON-SMOKERS: A COHORT STUDY OF 3.7 MILLION PEOPLE}

LJ Horsfall*, G Rait. Research Department of Primary Care and Population Health, UCL, London, UK

\subsection{6/jech-2020-SSMabstracts. 103}

Background There have been reports that lung cancer in nonsmokers (LCINS) is increasing in the UK but it is unclear whether this simply reflects fewer people smoking cigarettes or changing environmental risk factors such as increased emissions from domestic combustion. We examined UK-wide sociodemographic trends in the incidence of LCINS.

Methods We identified a cohort of 3,679,831 people selfreporting to their general practice physician as non-smokers from The Health Improvement Network (THIN) IQVIA ${ }^{\text {TM }}$ Medical Research Data. Using multivariable Poisson regression, we estimated gender-specific time-trends in the incidence of LCINS for 1998-2018 and explored the impact of geographic location, social deprivation and urban-rural classification.

Results The analysis included 3,121 cancer events and 16,051,244 person-years (PYs). Earlier time periods, high social deprivation, urban living, and residing in the North of England were associated with higher age-adjusted LCINS rates in men. Living in the North of England was the only clear risk factor for women. Between 1998-2008, age-adjusted rates in men declined by $9 \%$ per year (95\%CI: 7-11\%) from an estimated 5.6 to 1.5 per 10,000 PYs and then remained stable. These time trends for men were similar across sociodemographic variables. Between 1998 and 2007, incidence rates were stable for women at 1.3 per 10,000 PYs. However, for the least socially deprived rates increased by around 5\% per year (95\%CI: 2-9\%) from an estimated 1.3 per 10,000 PYs in 2008 to 2.1 in 2018.

Conclusion The incidence of LCINS has reduced or remained stable for most of the UK with the exception of women living in the least socially deprived areas.

\section{P08 THE PSYCHOSOCIAL DETERMINANTS OF QUALITY OF LIFE IN BREAST CANCER SURVIVORS: A SCOPING REVIEW}

${ }^{1} \mathrm{MG}$ Culbertson, ${ }^{1} \mathrm{~KB}$ Bennett, ${ }^{2} \mathrm{CK}$ Kelly, ${ }^{3} \mathrm{LS}$ Sharp, ${ }^{1} \mathrm{CC}$ Cahir* ${ }^{1}$ Division of Population Health Sciences, Royal College of Surgeons in Ireland, Dublin 2, Ireland; ${ }^{2}$ Mater Misericordiae University Hospital, Dublin 1, Ireland and Cancer Trials, Ireland; ${ }^{3}$ Population Health Sciences Institute, Newcastle University Centre for Cancer, Newcastle University, Newcastle, UK

\subsection{6/jech-2020-SSMabstracts. 104}

Background Breast cancer care today involves state-of-the-art biomedical treatment but can fail to address the broader psychosocial and quality-of-life (QoL) issues associated with the transition to breast cancer survivorship. This scoping review examines the evidence on the influence of psychosocial determinants on QoL in breast cancer survivors.

Methods Scoping review methodology was used to: (1) identify the research question(s); (2) identify relevant studies; (3) undertake study selection; (4) extract data; (5) collate, summarise and report the results.

Results A total of 34 studies met the inclusion criteria. The majority of studies were conducted in the US $(n=23,68 \%)$ and were mainly cross-sectional $(n=27,79 \%)$. Sixteen psychosocial determinants of QoL were identified. Social support $(n=16,47 \%)$, depression $(n=7,21 \%)$ and future appraisal and perspective $(n=7,21 \%)$ were the most frequently investigated determinants. Eighteen different QoL measures were used. A range of different measurement tools were also used per psychosocial determinant (weighted average $=6$ ). The 16 studies that measured the influence of social support on QoL employed 11 different measures of social support and 10 different measures of QoL. In general, across all 34 studies, a higher level of a positive influence and a lower level of a negative influence of a psychosocial determinant was associated with a better QoL e.g. higher social support and lower levels of depression were associated with a higher/better QoL. For some determinants such as spirituality and coping skills the influence on QoL varied, but these determinants were less commonly investigated.

Conclusion Consensus around measures of QoL and psychological determinants would be valuable and would enable research to determine the influence of psychosocial determinants on QoL adequately. Research in other healthcare settings beyond the US is required, in order to understand the influence of organisation and follow-up clinical and supportive care on psychosocial determinants and QoL and to improve the quality of care in breast cancer survivors.

\section{P09 DEVELOPMENT OF A COMMUNITY-BASED INTERVENTION TO INCREASE UPTAKE OF HOME BOWEL CANCER SCREENING IN SOUTH ASIANS: A MIXED METHODS STUDY}

${ }^{1} \mathrm{~L}$ Smith ${ }^{*}{ }^{2} \mathrm{~S}$ Howcutt, ${ }^{3} \mathrm{P}$ Saini, ${ }^{2,4} \mathrm{~J}$ Brett, ${ }^{2,4} \mathrm{C}$ Henshall, ${ }^{2,4} \mathrm{E}$ Watson. ${ }^{1} \mathrm{C}$ CAHR, Faculty of Health Sciences, University of Hull, Hull, UK; ${ }^{2}$ Faculty of Health and Life Sciences, Oxford Brookes University, Oxford, UK; ${ }^{3}$ School of Psychology Faculty of Health, Liverpool John Moores University, Liverpool, UK; ${ }^{4}$ OXINMAHR, Faculty of Health and Life Sciences, Oxford Brookes University, Oxford, UK

\subsection{6/jech-2020-SSMabstracts. 105}

Background Bowel cancer is common and accounts for $10 \%$ of all cancer mortality-second only to lung cancer deaths. If detected early through screening tests, mortality is significantly reduced. The NHS Bowel Cancer Screening Programme (BCSP) invites adults aged 60-74 years to carry out a home screening test biennially. The national target for test completion is 60\%; completion is substantially lower ( 30\%) amongst South Asian populations.

Universal approaches to increase screening uptake, e.g. text reminders, are effective, but may widen health inequalities, as they tend to benefit individuals with greater agency. Strategies tailored to ethnic minority groups show promise but are poorly specified and evidence of effectiveness is lacking. Limited evidence suggests that barriers to bowel cancer screening for South Asians are complex.

The aim was to develop a community-based intervention to increase completion of the home bowel screening test in South Asians. 